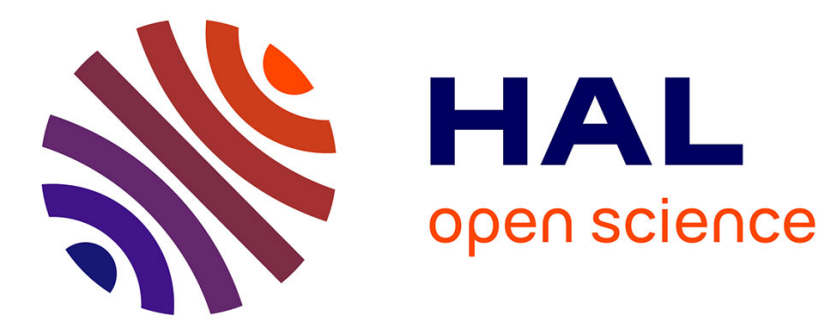

\title{
EBIC CONTRAST OF DEFECTS IN SEMICONDUCTORS
}

P. Wilshaw, T. Fell, M. Coteau

\section{To cite this version:}

P. Wilshaw, T. Fell, M. Coteau. EBIC CONTRAST OF DEFECTS IN SEMICONDUCTORS. Journal de Physique IV Proceedings, 1991, 01 (C6), pp.C6-3-C6-14. 10.1051/jp4:1991601 • jpa-00250673

HAL Id: jpa-00250673

https://hal.science/jpa-00250673

Submitted on 1 Jan 1991

HAL is a multi-disciplinary open access archive for the deposit and dissemination of scientific research documents, whether they are published or not. The documents may come from teaching and research institutions in France or abroad, or from public or private research centers.
L'archive ouverte pluridisciplinaire HAL, est destinée au dépôt et à la diffusion de documents scientifiques de niveau recherche, publiés ou non, émanant des établissements d'enseignement et de recherche français ou étrangers, des laboratoires publics ou privés. 


\title{
EBIC CONTRAST OF DEFECTS IN SEMICONDUCTORS
}

\author{
P.R. WILSHAW, T.S. FELL and M.D. COTEAU \\ Department of Materials, Parks Road, GB-Oxford OX13PH, Great-Britain
}

\begin{abstract}
The experimental requirements for relating EBIC contrast measurements to the recombination strength of defects in non-ideal specimens is discussed. Three criteria are given which must be met for such an interpretation of measurements to be made. In the second part of the paper the mechanism for recombination at dislocations in silicon is described together with experimental results from clean and decorated dislocations. Details are given of those situations in which copper contamination does not increase dislocation activity. Finally work on decorated stacking faults in silicon is presented.
\end{abstract}

\section{Introduction.}

The EBIC technique has been used for a long time to provide a spatial map of centres of recombination activity in semiconductor specimens [1]. The results obtained are normally presented in the form of micrographs where the dark regions correspond to fast recombination of carriers at defects such as dislocations or grain boundaries. More recently, however, it has become clear that many of the electronic properties of extended defects will only be understood if measurements of their properties are made with high spatial resolution [2]. Thus techniques such as EBIC, LBIC and CL are being increasingly used for quantitative study of extended defects. However it is not simple to relate measured values of contrast to materials properties of the defect such as, for example, the number of impurity atoms decorating a dislocation. The problem is two fold. In the first place it is necessary to relate the measured contrast of the defect to its efficiency as a recombination centre $\gamma$, and secondly to find a way of relating this recombination efficiency to the materials problems to be investigated. For the first part of the problem it is necessary to consider the spatial distribution of the generation of carriers in the specimen and their diffusion and recombination in the bulk together with their recombination at the defect concerned. In addition for a full solution the effect of carrier drift and altered recombination within the depletion region should also be taken into account. It is because of these effects that the "geometry" of the specimen ie. the relative positions of the defect, depletion region edge and its width together with the electron beam range are so important for accurate deduction of a defect's recombination strength from its measured contrast values. To a large extent these problems have now been solved theoretically using both analytical [3-5] and numerical [6,7] approaches. However it is the purpose of the first part of this paper to discuss ways in which the recombination efficiencies deduced from experiments can be in error due to the non-ideal nature of the specimens investigated, and in particular the effects that their electrical characteristics have on the EBIC contrast measured.

In the second part of the paper recombination at dislocations in silicon will be described for which a model is available giving an excellent fit with experimental results. Using this model it is then possible to obtain materials information about the defects studied. In the final section recombination at defects for which no adequate model yet exists is discussed.

\section{Experimental Considerations for Accurate EBIC Contrast Measurements}

Although the work discussed here refers specifically to EBIC much of it is also applicable to the LBIC technique since the diffusion and recombination of carriers and the measurement of the induced signal take place take place in the same way as for EBIC. The difference is in the form of the generation function which is now due to a light beam 
rather than an electron beam and in this case the spatial distribution of generated carriers is changed by changing the wavelength of the incident light.

\subsection{Specimen Considerations.}

Quantitative EBIC experiments of defects often take the form of measuring the defect contrast as a function of temperature and sometimes also as a function of the incident beam current $[8,9]$. The observed changes in contrast are then taken to depend on changes in the recombination strength of the defect. However such a direct relationship between the experimentally measured contrast and the defect recombination strength is not always valid because in some cases the non-ideal nature of the specimens and their electrical contacts will affect the signal measured. In the following a brief analysis is given of those considerations which should be taken into account when attempting to make accurate EBIC contrast measurements. A more complete description of this work can be found in [10].

It has been shown [3] that for an "ideal" EBIC specimen the contrast $\mathrm{C}$ of a point or line defect can be expressed as:

$$
C=f(\gamma) \cdot g(R, d, w, L)
$$

where $g$ is a function dependent on $R$ the range of the incident electrons, $d$ and $w$ are parameters describing the defect position and the depletion region width associated with the collecting junction and $L$ is the minority carrier diffusion length. The form of $\mathrm{g}$ depends on the specimen geometry chosen. $\gamma$ is a measure of the defect recombination strength and for a "weak" defect which only slightly perturbs the minority carrier concentration $f(\gamma)=\gamma$. Clearly for the observed changes to be attributed to changes in the recombination strength of the defect, the parameters in the function $\mathrm{g}$ must remain constant. In practice this means ensuring that the depletion region width does not change significantly and thus any bias voltage applied to the collecting junction must not alter in the course of an experiment irrespective of whether the junction or the specimen ohmic contact characteristics change. Secondly the diffusion length must be checked experimentally to determine that it is constant throughout an experiment. Should the diffusion length change, a direct relationship between $\mathrm{C}$ and $\gamma$ is no longer possible. Although even in these circumstances if CL measurements are available it is possible, with extra effort, to use a combined EBIC/CL technique developed by Jacobowicz [11] to deduce changes in $\mathrm{g}$ from changes in the measured contrast independent of the changes in $\mathrm{L}$.

In many EBIC investigations the specimens used are far from ideal in that not all the induced current generated by the incident beam actually flows through the external measuring circuit. In such a case the problem of the experimental measurement of the EBIC signal generated has to be further considered. An EBIC specimen used to generate a current when no bias is applied can be modelled by the circuit shown in figure 1 .

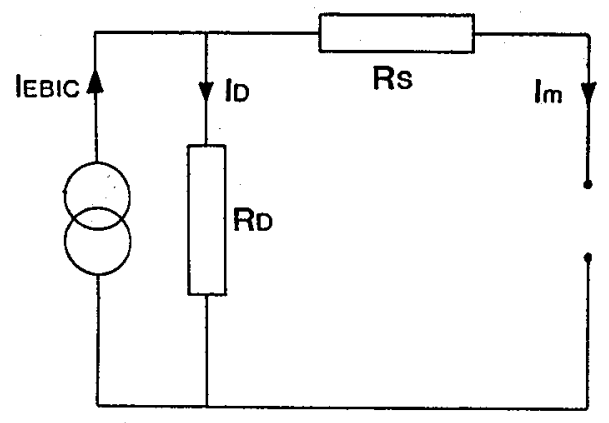

Figure 1. Equivalent circuit for EBIC measurement. $R_{\mathrm{s}}$ is the series resistance comprising the specimen,ohmic contact and measuring circuit resistance. $R_{d}$ is the forward biased resistance of the EBIC collecting junction. $I_{m}$ and $\mathrm{I}_{\text {EBIC }}$ are the measured and ideal EBIC signals respectively.

The induced current can be considered to be generated by a perfect current source in parallel with the resistance $R_{d}$ of the diode of the collecting junction and in series with a resistance $R_{\mathrm{s}}$ comprised of the resistance of the specimen itself, the ohmic contact to the specimen and the input resistance of the EBIC current amplifier which will usually be negligible. The "ideal" EBIC signal $I_{\text {EBIC }}$ flows partly through the diode $I_{d}$ without being measured and also through the series resistance and hence the measuring circuit to give a measured current $\mathrm{I}_{\mathrm{m}}$. The measured current is thus $I_{m}=\left[R_{d} /\left(R_{d}+R_{s}\right)\right]$. $I_{E B I C}$. The diode resistance can be obtained by considering $I_{d}$ the current flowing the diode which varies according to: 


$$
\mathrm{I}_{\mathrm{d}}=\mathrm{I}_{0}\left[\exp \left(q \mathrm{~V}_{\mathrm{d}} / \beta \mathrm{kT}\right)-1\right]
$$

where $V_{d}$ is the voltage across the junction, $\beta$ is the ideality factor; usually between 1 and 2 and $I_{0}$ is the diode saturation current. In many EBIC experiments with collecting junctions of surface area of the order of square millimetres and with defects within them, values for $I_{0}$ may be as large as $10^{-7}$ or $10^{-6}$ A whilst $I_{\text {EBIC }}$ may be as small as $10^{-8}$ or $10^{-9} \mathrm{~A}$. For these conditions the exponential term in equation 2 can be expanded and higher order terms neglected to give $R_{d}=V_{d} \Lambda_{d}=\beta k T / q I_{0}=R_{0}$. This resistance $R_{0}$ is ohmic and dependent on $I_{0}$ but not on the induced current flowing through the diode. For $I_{d} \gg I_{0}$ equation (2) may be simplified to its more usual form $I_{d}=I_{0} \exp \left(q V_{d} / \beta k T\right)$ and for these conditions the diode resistance, now written $R_{1}$, is much smaller than $R_{0}$ and will show the typical non-ohmic, forward biased diode junction characteristics.

From this simple circuit analysis it can be seen that there are three regions of behaviour for the EBIC signal measured from a non-ideal specimen:

1) $R_{s} \ll R_{d}$. The measured EBIC signal $I_{m}$ is equal to the ideal signal $I_{\text {EBIC }}$

2) $R_{s}>R_{d}$ and $I_{d}<I_{0}$ so that $R_{d}=R_{0}$. Thus $I_{m}=\left[R_{0} /\left(R_{0}+R_{s}\right)\right] . I_{\text {EBIC. }}$. The measured signal $I_{m}$ is now less than $\mathrm{I}_{\mathrm{EBIC}}$. However it is directly proportional to $\mathrm{I}_{\mathrm{EBIC}}$ and any contrast measurement will be equal to the ideal contrast of the defect. Thus provided the parameters in the function $\mathrm{g}$ of equation 1 remain constant, both regime 1 and 2 may be used for accurate EBIC contrast measurements in which the measured change in contrast with experimental parameters, such as temperature, can be directly related to changes in the defect recombination strength.

3) $R_{s}>R_{d}$ and $I_{d}>I_{0}$ so that $R_{d}=R_{1}$. The measured current is again reduced but is no longer proportional to $I_{\text {EBIC }}$ because $R_{1}$ varies with $I_{d}$ the current flowing through the forward biased junction. This regime is unsuitable for EBIC contrast measurements with the measured signal now approximating to an electron beam induced voltage (EBIV) signal. In this regime it can be shown [10] that the measured contrast decreases with increasing $\mathrm{I}_{\mathrm{EBIC}}$.

Thus in order to interpret experimental EBIC results in terms of $\gamma$ it is necessary to establish whether signal collection is in this third regime. This can be done experimentally by measuring the ratio $I_{m} / I_{\text {beam. }}$. Since $I_{\text {EBIC }}$ is generally proportional to $I_{\text {beam }}$ then for regimes 1 and 2 this ratio is constant whereas for regime 3 it decreases with increasing $\mathrm{I}_{\text {beam }}$. Figure 2 shows values for the ratio $\mathrm{I}_{\mathrm{m}} / \mathrm{I}_{\text {beam }}$ at different temperatures and beam currents for a plastically deformed piece of $n$-type silicon with readings obtained from a region of the specimen free of dislocations. The arbitrary units are the same for each graph. At $300 \mathrm{~K} \mathrm{I} / I_{\text {beam }}$ is independent of $\mathrm{I}_{\text {beam }}$ for all beam currents but as the temperature is reduced the shape of the graph changes. At $200 \mathrm{~K}$ the ratio of $I_{\mathrm{m}} I_{\text {beam }}$ is smaller indicating that $R_{s}>R_{d}$ and so $I_{m}<I_{\text {EBIC. }}$. Also at beam currents approaching $10^{-9} \mathrm{~A}$ the ratio decreases quickly with increasing $\mathrm{I}_{\text {beam }}$ showing that at this temperature and beam current regime 3 has been entered. At $131 \mathrm{~K}$ the ratio is further reduced showing $R_{s}$ has increased still further and $I_{m} / I_{\text {beam }}$ now starts getting smaller with increasing beam current at approximately $10^{-10}$ A showing that $I_{d}>I_{0}$ for smaller values of $I_{\text {beam }}$ than at $200 \mathrm{~K}$. These results are consistent with a series resistance, due mainly to the ohmic contact, which rises as the specimen is cooled [12]. Indeed at $131 \mathrm{~K} R_{s}$. was measured to be $>30 \mathrm{k} \Omega$ and thus at these low temperatures regime 3 for this specimen is easily obtainable at beam currents frequently used for EBIC investigations.
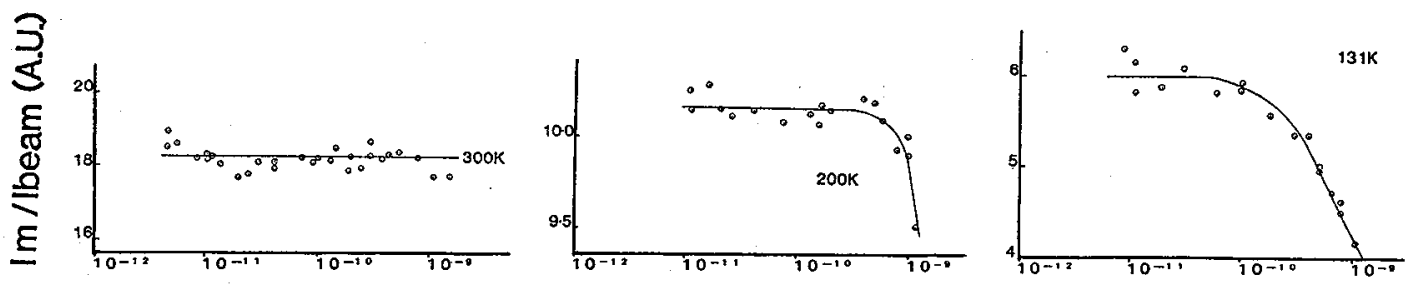

\section{Ibeam (A)}

Figure 2. Measured values of $\mathrm{I}_{\mathrm{m}} / \mathrm{I}_{\text {beam }}$ at different temperatures for an n-type Si specimen. Decreasing values of $\mathrm{I}_{\mathrm{m}} /_{\text {beam }}$ as $\mathrm{I}_{\text {beam }}$ increases indicate $\mathrm{I}_{\mathrm{D}}>\mathrm{I}_{0}$. 
Figure 3 shows EBIC contrast measurements from a dislocation in the same specimen as that used to obtain the data in figure 2. From this it can be seen that the contrast begins to decrease more rapidly at large beam currents and low temperatures as regime 3 is entered for this specimen. The experimental data from this part of the graph is thus an artefact of the electrical characteristics of the specimen and does not represent actual changes in the recombination efficiency of the dislocation. Without such an independent measurement of $I_{m} / I_{\text {beam }}$ it is doubtful that such a conclusion could have been reached.

Figure 3. EBIC contrast measurements of a dislocation corresponding to the data in fig. 2. The large decrease in EBIC contrast at low temperatures and high beam currents occurs when $I_{D}>I_{0}$ as indicated in fig. 2 .

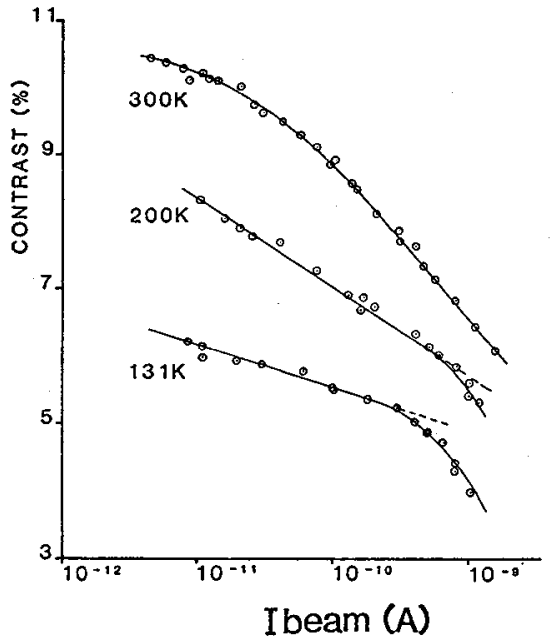

Although a series resistance of $>30 \mathrm{k} \Omega$ may seem very high, such values can be obtained when low doped specimens are investigated [13] or when an ohmic contact is fabricated on a specimen for which it is not possible to give the contact an anneal without disturbing the defect structure to be investigated. However, whilst this analysis indicates that there may be problems associated with large values of series resistance it also shows that accurate EBIC or LBIC contrast measurements may still be obtained for very high $R_{s}$ provided $I_{d}<I_{0}$.

In summary the experimental requirements that the measured EBIC contrast and its changes can be directly related to the defect recombination strength during an experiment are i) that the depletion region width does not change, ii) that the minority carrier diffusion length remains constant and iii) that the ratio $\mathrm{I}_{\mathrm{m}} / \mathrm{I}_{\text {beam }}$ is independent of $\mathrm{I}_{\text {beam }}$ for all experimental conditions used. If this is so then specimens with a large series resistance can still be employed for accurate measurements.

\subsection{Analysis of the Absolute Magnitude of Defect Contrast.}

The purpose of this section is to illustrate the difficulties associated with inferring information about the defect recombination strength from a single EBIC contrast measurement and when making comparisons between different defects in, perhaps, different specimens with a view to obtaining information about their relative recombination strengths. The difficulty arises because the contrast measured is a strong function of the parameters $R, d$, $w$ and also, particularly for short diffusion lengths, $\mathrm{L}$. Thus a direct comparison between different defects or information on the absolute value of $\gamma$ can only be inferred if all these parameters are known with some accuracy. In some situations, for example when dislocations are inclined to the surface at a known angle this can be the case, and then information can then be obtained from the absolute level of contrast and the shape of the defect image produced [6,7]. But in many experiments this is not so and the problem is further compounded because, as is shown in the next section, the recombination strength of a given type of defect can depend on the doping concentration of the material and also the temperature and beam current used to measure it. Thus measurement of different values of $\gamma$ between different defects does not necessarily imply differences in the nature of the defects themselves, rather it could be due to changes in their environment and measuring conditions.

Figure 4(a-c) shows the additional ambiguity introduced when comparing images of defects. The figure shows EBIC images of the same misfit dislocation network, contaminated with stainless steel, lying $1.6 \mu \mathrm{m}$ below a surface Schottky barrier in n-type $6.6 \times 10^{15} \mathrm{~cm}^{-3} \mathrm{Si}_{94} \mathrm{Ge}_{06}$ examined using a beam current of $2 \times 10^{-11} \mathrm{~A}$. Figure $4 \mathrm{~b}$ has been taken with the settings of gain and DC offset applied to the EBIC signal so that a given contrast defect produces 4 times the voltage swing at the output of the EBIC electronics compared to those used in figure 4a. In figure $4 \mathrm{c}$ the same amplifier settings as in figure $4 \mathrm{a}$ have been used but the brightness and contrast settings for the SEM photo-display monitor were changed. Clearly the actual processing of the EBIC signal can result in very different images being obtained and for this reason, where ever possible, a quantitative measure of contrast in the image should be given. 
Figure 4(d-f) shows images of misfit dislocations in the same material as figure $4(a-c)$, but with different specimen processing and with different amounts of material polished from the surface. In figure $4 \mathrm{~d}$ the dislocations lie $1.25 \mu \mathrm{m}$ deep and were examined using $15 \mathrm{kV}$ electrons and the amplifier settings of figure $4 \mathrm{~b}$ which are sensitive to low contrast levels. Even with these amplifier settings little activity is apparent. In figure $4 \mathrm{e}$ the specimen has been polished so that the dislocations are now $0.5 \mu \mathrm{m}$ deep. The amplifier settings are now the same as for figure $4 \mathrm{a}$ and are less sensitive to contrast. The image in $4 \mathrm{f}$ is taken under the same conditions as $4 \mathrm{e}$ except that a $25 \mathrm{kV}$ accelerating voltage is used. The dislocations now show strong activity. Such a series of micrographs illustrate the difficulty in accurately judging the activity of defects and show that if a comparison between defects is to be made the experimental conditions should be the same in each case.

\section{Misfit Dislocations Located at a SiGe/Si MBE Grown Interface}

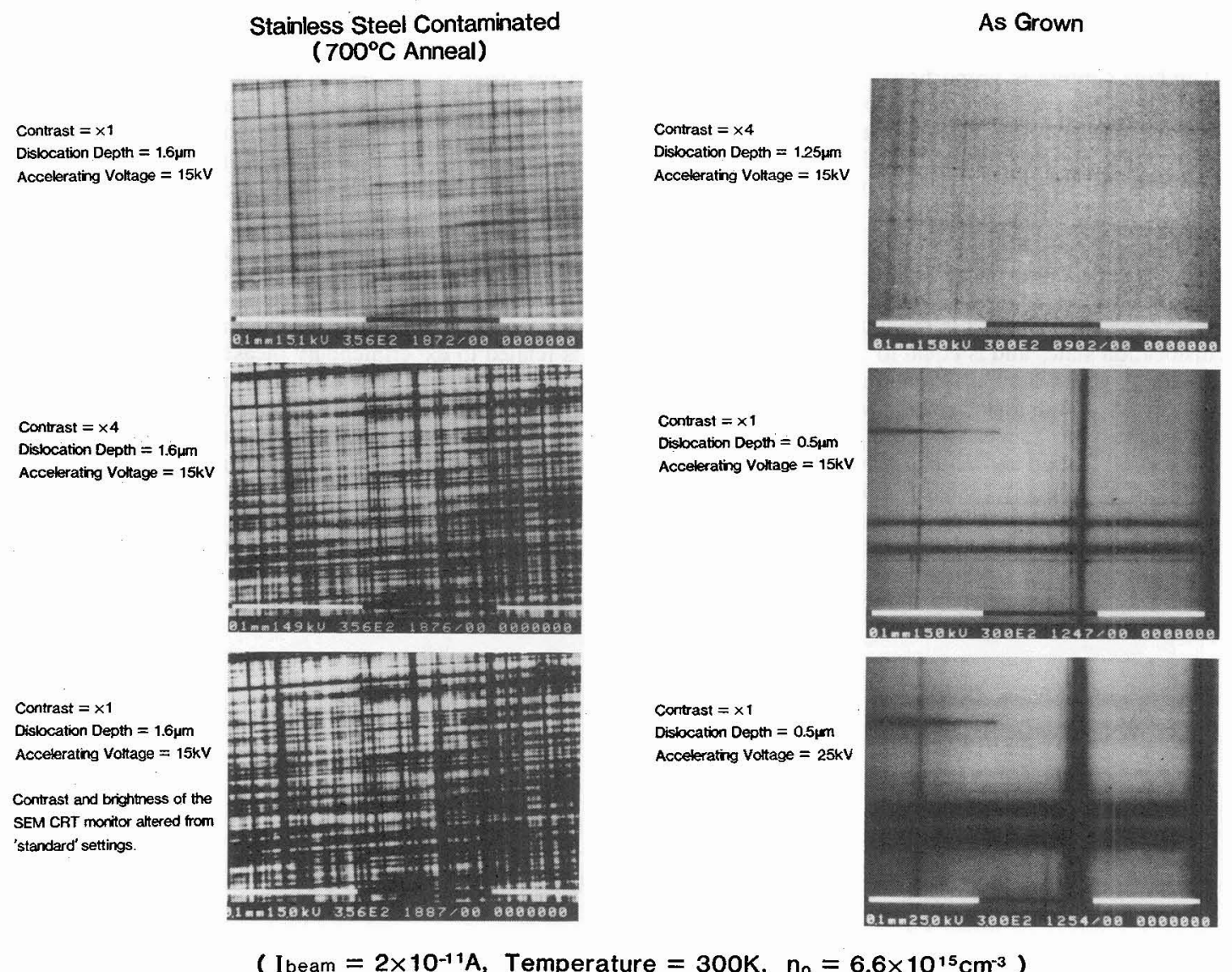

Figure 4. EBIC micrographs of misfit dislocations at a $\mathrm{Si} \mathrm{Si}_{0.94} \mathrm{Ge}_{.06}$ interface showing how different electronic processing (a-b), photographic conditions (a-c), dislocation depth (d-e), and electron beam accelerating voltage (e-f) can effect the images obtained. 


\section{Recombination at Dislocations in Silicon.}

\subsection{Recombination Mechanism.}

Recombination of electron hole pairs in silicon has been analysed in terms of the capture of electrons and holes being controlled by the charge trapped at the dislocation and hence by the band bending surrounding the dislocation $[8,14,15]$. In this model the electron capture is described by thermal activation of the carriers over the potential barrier of the bent bands which for n-type material is given by:

$$
\mathrm{Je}=\mathrm{C}_{\mathrm{e}} \mathrm{N}_{\mathrm{d}} .\left[(1-\mathrm{f}) \mathrm{n}_{0} \exp (-q \phi / \mathrm{kT})-\mathrm{fN}_{\mathrm{c}} \exp \left(-\mathrm{q} \phi_{0} / \mathrm{kT}\right)\right]
$$

where $\mathrm{J}_{e}$ is the net electron capture rate per unit dislocation length, $\mathrm{C}_{\mathrm{e}}$ is the probability of the transition of an electron in the conduction to a dislocation state, $N_{d}$ is the number of dislocation states per unit dislocation length, $N_{c}$ is the effective density of states in the conduction band, $\mathrm{n}_{0}$ is the free carrier concentration and $\mathrm{f}$ is the occupancy factor of the dislocation states. $\phi$ and $\phi_{0}$ are, respectively, the potential barriers for excitation of free electrons over the bent conduction bands into the dislocation states and for trapped electrons out of the dislocation states and into the conduction band. Since the band bending $\phi$ decreases with decreasing dislocation line charge $Q$, equation 2 shows that electron capture increases with decreasing line charge.

The hole capture is proportional to the cross-sectional area of the space charge region surrounding the dislocation and hence increases for increasing dislocation charge. At steady state the rate of electron and hole capture at the dislocation must be equal and consequently it is possible to obtain an expression for the recombination strength of a dislocation by solving together the expressions for electron and hole capture. In addition using equation (1) it is possible to relate recombination at the dislocation to the experimentally observed EBIC contrast $[14,15]$ to give:

$$
C=\frac{\alpha T}{n_{0}} \ln \left[\frac{(1-f) n_{0} C_{e} N_{d}}{J+R}\right]
$$

where $\alpha$ is a constant, $\mathrm{J}$ is the total recombination flux and $\mathrm{R}$ is the thermal re-emission rate of electrons from the dislocation states and is equal to $\mathrm{fN}_{\mathrm{c}} \mathrm{C}_{\mathrm{e}} \mathrm{N}_{\mathrm{d}} \cdot \exp \left(-\mathrm{q} \phi_{0} / \mathrm{kT}\right)$. J is related to experimentally measurable parameters by $\mathrm{J} \sim \eta \mathrm{I}_{\text {beam }} \mathrm{C} / \mathrm{ql}$ where $\eta$ is the number of electron hole pairs generated for each electron in the incident beam and $\mathrm{l}$ is the length of the dislocation at which recombination takes place. The excess minority carrier concentration and hence J can be changed by altering the beam current used for an experiment. A more detailed analysis of this model for recombination shows that providing that the factors in the function $g$ of equation (1) remain constant during an experiment then any changes in dislocation contrast are proportional to changes in the dislocation charge. Figure 5 shows theoretical plots of dislocation contrast versus temperature with the parameters such as dislocation energy level position, and the concentration of defect states $\mathrm{N}_{\mathrm{d}}$, chosen to illustrate the different regimes of behaviour on two curves. In the first regime at low temperatures or large excess minority carrier concentrations the dislocation contrast is approximately proportional to temperature for constant beam current and varies as $-\ln \left(\mathrm{I}_{\text {beam }}\right)$ at constant temperature.

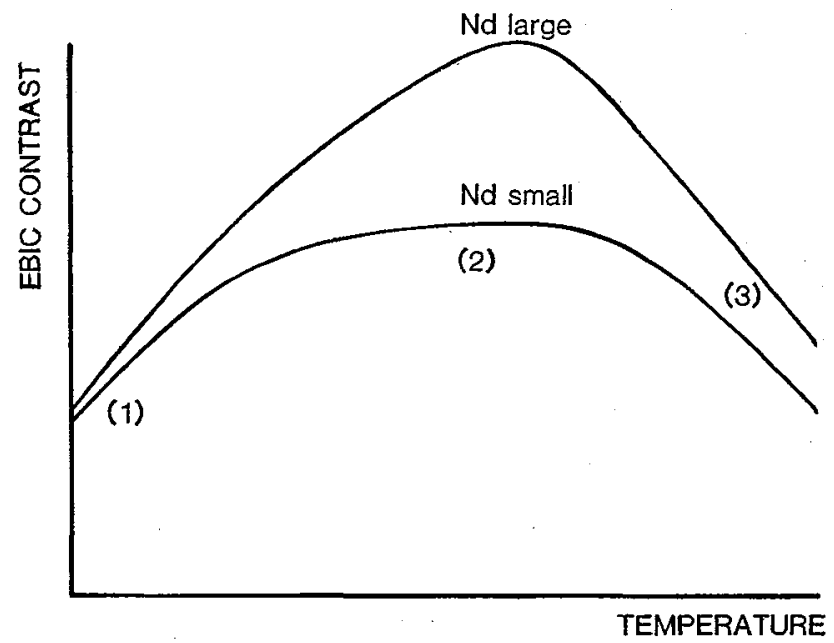

Figure 5. Theoretical curves of EBIC dislocation contrast versus temperature. 
This behaviour may be understood by considering a dislocation initially at equilibrium with line charge $Q$. If an excess minority carrier concentration is introduced by the incident beam, the hole capture rate is increased. However the rate of capture of electrons is initially unchanged because $\Delta \mathrm{n} \ll \mathrm{n}_{0}$ for the conditions considered here. Thus $\mathrm{J}_{\mathrm{h}}>\mathrm{J}_{\mathrm{e}}$ which will result in the magnitude of the negative dislocation charge decreasing from its equilibrium value. As $Q$ decreases the electrostatic barrier to electron capture also decreases and thus $J_{e}$ increases according to equation 3. Reducing $Q$ also decreases $J_{h}$ and this process will continue until $J_{e}=J_{h}$ and the steady state is reached. Thus for increasing hole concentrations the dislocation charge has to be progressively decreased such that the extra value of the electron capture matches the increased hole capture. This reduction in dislocation charge leads in turn to a reduction in i) the space charge region surrounding the dislocation, ii) the efficiency of the dislocation as a centre for hole recombination and iii) the EBIC contrast produced by the defect, the contrast changing as $-\ln \left(I_{\text {beam }}\right)$ in this regime. By a similar argument if the temperature is increased, thermal activation of the electrons over the potential barrier is also increased and so a given capture rate is achieved with a higher line charge so leading to $\mathrm{C}$ increasing linearly with temperature. In this regime $\mathrm{J}$ the electron capture is much greater than $\mathrm{R}$ the thermal re-emission.

As the temperature is increased further or the excess minority carrier concentration is reduced the charge on the dislocation initially in regime 1 will increase and tend to approach the equilibrium charge it would have had in the absence of recombination. If the concentration of states at the dislocation $N_{d}$, and the position of the Fermi level are such that at equilibrium all the states are occupied and the dislocation energy level is below the Fermi level, then the recombination behaviour lies in regime 2 . In this regime, in which the dislocation charge is close to equilibrium, $\mathrm{f}$ is nearly unity and so $Q$ is nearly constant and thus the recombination strength is approximately independent of both temperature and beam current.

If however, the concentration of states at the dislocation is such that the band bending is sufficient for the dislocation level to intersect the Fermi level at values of $\mathbf{f}<1$, then at equilibrium the dislocation level will be pinned to the Fermi level. Thus, regime 3 occurs when the temperature is high enough and the excess minority carrier concentration low enough that the dislocation energy level is pinned to the Fermi level. In this regime the recombination strength decreases with increasing temperature as the Fermi level moves towards the centre of the band gap. The recombination strength is however independent of the minority carrier concentration. For these conditions $f<1$ and $R>J$.

\subsection{Interpretation of EBIC Contrast Measurements}

In the following an analysis is given that shows how such information as the concentration of dislocation states $\mathrm{N}_{\mathrm{d}}$ and the position of these states $\phi_{0}$ may be obtained from a series of dislocation contrast measurements as a function of temperature and beam current.

The EBIC contrast of a dislocation is a measure of $Q$ and hence $\phi$ the band bending since the two are easily related [16]. Thus, as described above, if the beam current in an experiment is increased so that the dislocation charge moves away from equilibrium (regime 1) then the EBIC contrast will decrease as the dislocation charge is decreased. Alternatively the recombination rate, which is equal to the net capture rate of electrons at steady state, can be viewed as having a straight forward thermally activated behaviour with activation energy $q \phi$ in regime 1 where thermal reemission is negligible. In this regime however the band bending $\phi$ is not necessarily constant but depends on the recombination taking place. Thus if the electron beam current is changed in the course of an experiment at different temperatures such that the measured EBIC contrast stays constant then it follows that the dislocation charge and hence band bending will also remain constant. Thus if the recombination flux at a dislocation $J$, which is equal to the net capture rate $\mathrm{J}_{e}$, is measured as a function of temperature whilst constant contrast is maintained then an Arrhenius plot of $\ln (J)$ versus $1 / T$ will yield the activation energy $q \phi$ for electron capture according to equation 3 with negligible thermal re-emission. In this case the activation energy measured will be that due to the band bending $\phi$ of the dislocation corresponding to that particular line charge $Q$ which gives rise to the particular value of contrast that was held constant for the dislocation investigated.

Figure 6 shows typical Arrhenius plots for a $60^{\circ}$ dislocation in n-type $10^{15} \mathrm{~cm}^{-3}$ silicon deformed at $420^{\circ} \mathrm{C}$. From these measurements of $\phi$ it can be seen that the band bending decreases as the contrast of the dislocation decreases. Extrapolation of the curves to $1 / \mathrm{T}=0$ results in their intersection as expected from equation 3 . Thus if sufficient Arrhenius plots are obtained for a particular dislocation, its contrast can be "calibrated" in terms of the band bending and hence the dislocation charge for each value of contrast. In this way measurement of the equilibrium dislocation contrast in regime 2 or 3 can then be used to deduce the equilibrium dislocation charge. Recalling that regime 2 obtains when all the states are full and beneath the Fermi level, quantitative analysis of the dislocation charge in this regime will determine the concentration of states $N_{d}$ and establish that the position of the states $\phi_{0}$ must be below the Fermi level at the temperatures measured. Conversely for dislocations demonstrating regime 3 behaviour quantitative analysis reveals $\phi_{0}$ but can only establish a lower limit for $\mathrm{N}_{\mathrm{d}}$ the number of states available for occupation. 


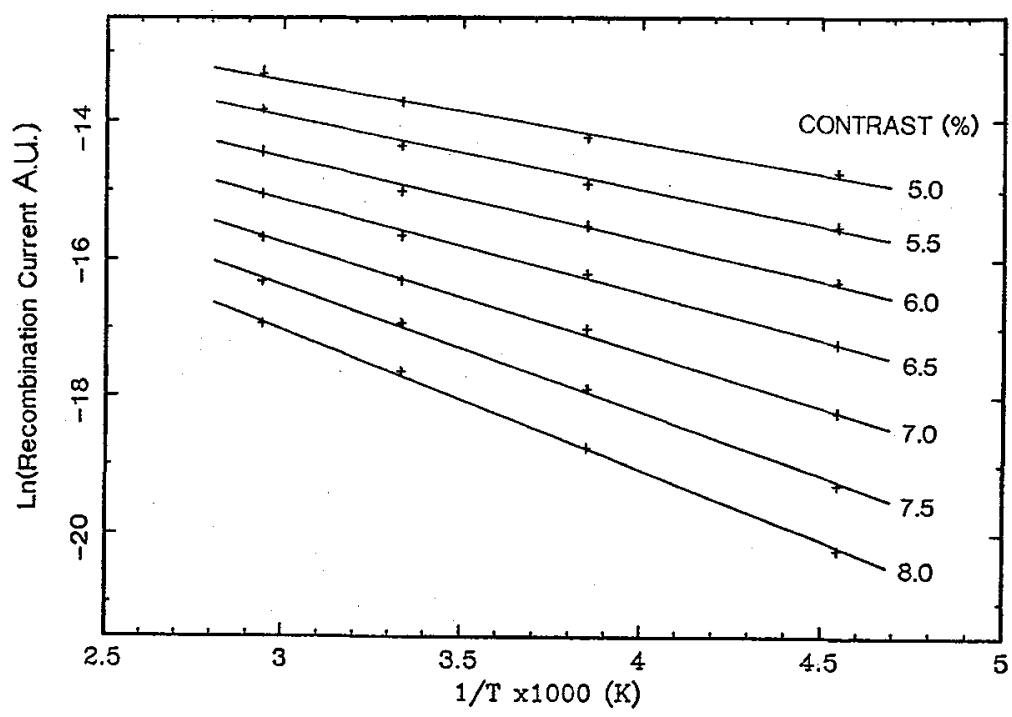

Figure 6. Arrhenius plots of recombination current versus temperature showing different activation energies for electron capture.

It is noted here in passing that since the band bending and dislocation charge at a dislocation is limited to the range from zero to the point at which the dislocation defect states intersect the Fermi level then so also is the dislocation contrast restricted to a similarly small range. For example a dislocation at a depth where it shows maximum contrast using a $15 \mathrm{kV}$ electron beam produces a contrast of $\sim 9 \%$ with $0.15 \mathrm{~V}$ band bending in $10^{15} \mathrm{~cm}^{-3}$ material [15]. This can be produced by a state $\sim 0.45 \mathrm{~V}$ below the conduction band pinned to the Fermi level at $300 \mathrm{~K}$ and consequently even the deepest states found at dislocations $(\sim 0.65 \mathrm{~V}$ deep) would produce peak contrast levels of $\sim 15-18 \%$ at $300 \mathrm{~K}$ in this material. However if the dislocation is decorated with precipitates or surrounded by extended point defect atmospheres then much higher contrast values can be obtained as shown in section 5 .

\section{Experimental.}

Deformation induced dislocations in n-type $10^{15} \mathrm{~cm}^{-3}$ silicon both with and without intentional contamination have been examined using an EBIC system based around a Philips 505 SEM which is similar to one described previously [17]. The specimens were prepared from ingots deformed using two stage compression along a [213] axis [18,15] with a pre-deformation at $850^{\circ} \mathrm{C}$ or $\sim 950^{\circ} \mathrm{C}$ to nucleate dislocations and a main deformation at $650^{\circ} \mathrm{C} \mathrm{or} 420^{\circ} \mathrm{C}$ to produce hexagonal dislocation loops lying on (111) planes with loop diameters up to $\sim 200 \mu \mathrm{m}$. EBIC specimens cut from the ingots deformed both by Prof. Alexander and co-workers in Koeln and by the present authors have been examined. Some of the ingots deformed in Oxford were intentionally contaminated by gently wiping once with a copper wire immediately prior to the main deformation

\subsection{Results From $420^{\circ} \mathrm{C}$ and $650^{\circ} \mathrm{C}$ Dislocations.}

Quantitative analysis of straight screw and $60^{\circ}$ dislocations produced by deformation at $420^{\circ} \mathrm{C}$ demonstrated relatively high levels of recombination activity with peak EBIC contrast values of $\sim 12 \%$ being measured with an accelerating voltage of $15 \mathrm{kV}$. A typical contrast versus beam current curve is shown in figure 7 . These dislocations were found to demonstrate regime 1 and 2 type behaviour. Following the analysis described above to generate Arrhenius plots to deduce the band bending of the dislocations the activity was found to be caused by a deep defect state $>0.52 \mathrm{~V}$ from the conduction band edge with $\mathrm{N}_{\mathrm{d}}$, the concentration of occupied states $\sim 2.9 \times 10^{6} \mathrm{~cm}^{-1}$. Identical behaviour was observed from specimens produced by the Oxford group and Koeln groups who have employed different starting material and deformation apparatus to produce the specimens. This provides some evidence that impurity contamination is not the source of the activity observed since this would inevitably be different in the different specimens. In addition, intentional introduction of impurities through incomplete cleaning procedures did not show an increase in activity that would be consistent with the $420^{\circ} \mathrm{C}$ activity being due to impurities. 


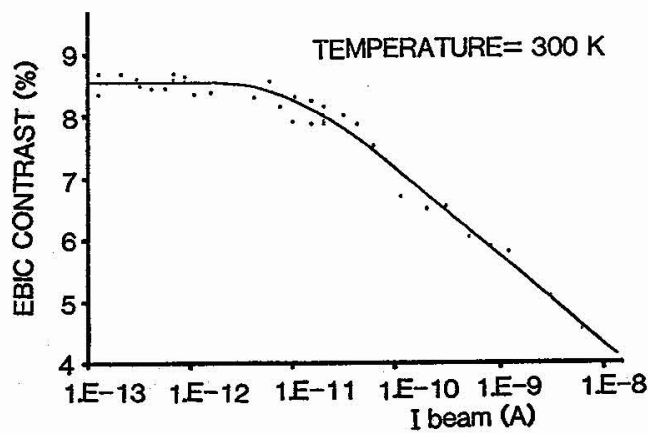

Figure 7. EBIC dislocation contrast versus beam current for a $420^{\circ} \mathrm{C}$ dislocation.

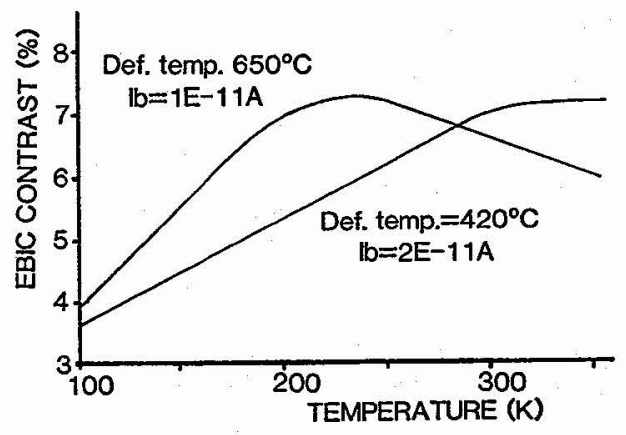

Figure 8. EBIC dislocation contrast versus temperature for $420^{\circ} \mathrm{C}$ and $650^{\circ} \mathrm{C}$ uncontaminated dislocations.

The straight screw and $60^{\circ}$ dislocations produced by deformation at $650^{\circ} \mathrm{C}$ showed much lower levels of electrical activity than their $420^{\circ} \mathrm{C}$ counterparts, with peak contrast values of typically $<2 \%$ being obtained with a $15 \mathrm{kV}$ beam. Typical contrast versus temperature curves for $420^{\circ} \mathrm{C}$ and $650^{\circ} \mathrm{C}$ dislocations are shown in figure 8 . These dislocations showed both regime 1 and 3 behaviour, ie. the dislocation states become pinned to the Fermi level as the Fermi level moves towards the centre of the gap. Quantitative analysis of these results found the dislocation states responsible for this activity to have an occupation limit $0.34-0.37 \mathrm{~V}$ below the conduction band edge and to have a concentration of $>5 \times 10^{5} \mathrm{~cm}^{-1}$. Because of the range of temperatures and doping used for these experiments, states shallower than $\sim 0.3 \mathrm{~V}$ could not be detected as they would be uncharged.

It is worth noting that analysis of EBIC contrast measurements from curved segments of dislocations where the radius of curvature is $\sim 0.5 \mu \mathrm{m}$ and hence with a dislocation kink density much higher than $\sim 5 \times 10^{5} \mathrm{~cm}^{-1}[19,15]$ show no increase in the number of defect states from the $\sim 5 \times 105 \mathrm{~cm}^{-1}$ found at straight dislocations. This indicates that the dangling bonds at the kinks must be largely reconstructed such that if any state is introduced into the band gap it is shallower than $\sim 0.3 \mathrm{~V}$ from the conduction band edge.

\subsection{Results Comparing $420^{\circ} \mathrm{C}$ and $650^{\circ} \mathrm{C}$ Copper Decorated Dislocations.}

Copper decorated $650^{\circ} \mathrm{C}$ deformation induced dislocations were found to show considerably higher activity than the uncontaminated $650^{\circ} \mathrm{C}$ dislocations with typical peak contrast levels of $\sim 8 \%$ being found. A typical image is shown in figure 9. Quantitative analysis found a new level present at these dislocations with an occupation limit 0.48 $0.51 \mathrm{~V}$ below the conduction band edge. However investigation of copper contaminated $420^{\circ} \mathrm{C}$ dislocations showed behaviour identical with the uncontaminated dislocations again giving values for $\mathrm{N}_{\mathrm{d}} \sim 2.9 \times 10^{6} \mathrm{~cm}^{-1}$ and $\mathrm{f}_{0}>0.52 \mathrm{~V}$. In no case did the copper decorated dislocations studied here show any evidence for precipitation either in EBIC images nor by etching experiments. However there was evidence for copper colonies being formed, in some cases adjacent to the dislocations studied in this work.

Figure 9. EBIC image of copper decorated, deformation induced dislocations induced at $650^{\circ} \mathrm{C}$.

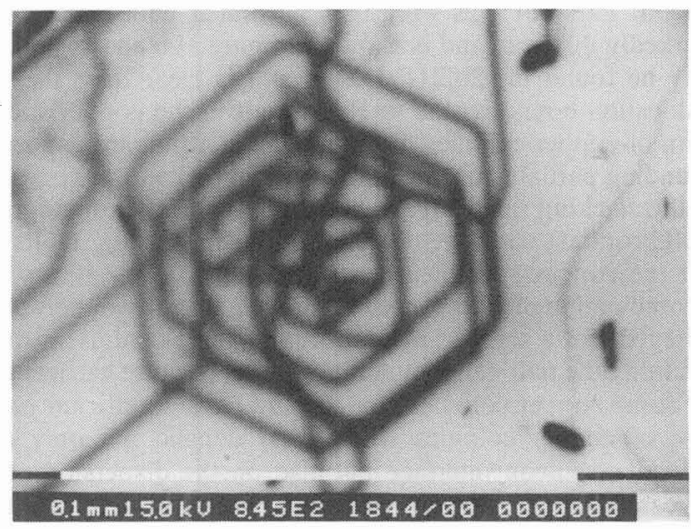




\subsection{Discussion.}

The dislocations showing least electrical activity in this work were the $650^{\circ} \mathrm{C}$ deformed dislocations without intentional contamination. The states at these dislocations are sufficiently shallow that they are readily pinned to the Fermi level with only a small dislocation charge. Decorating these dislocations with copper introduced a deeper level in sufficient concentration that this was also pinned to the Fermi level but with increased band bending and hence increased dislocation charge. The effect of this deeper copper level becoming pinned is to raise the original shallow $650^{\circ} \mathrm{C}$ states above the Fermi level thus rendering it essentially inactive as a recombination centre and also we assume, undetectable by techniques such as DLTS.

$420^{\circ} \mathrm{C}$ deformation produces a small number of states such that all are occupied without them being pinned to the Fermi level. The band bending this produces is sufficient to raise any shallower states associated with the dislocations, including the copper impurity level if present, above the Fermi level thus making them inactive as recombination centres. This accounts for the similar results obtained from $420^{\circ} \mathrm{C}$ dislocations with differing amounts of contamination and shows that the commonly held view that the higher the concentration of impurities at dislocations the higher the recombination activity, is not always correct. This model of dislocation activity however only remains valid provided the impurities do not precipitate at the dislocation or form extended point defect atmospheres. It should be noted that a qualitative analysis of these results may have led to the conclusion that copper impurity atoms are gettered to $650^{\circ} \mathrm{C}$ dislocations but not to $420^{\circ} \mathrm{C}$ dislocations which then may have pointed to some fundamental difference between the two dislocation types. However we believe this is not the case and the full quantitative analysis provides a simpler explanation based on the electronic properties of the copper impurities and the defect states at $420^{\circ} \mathrm{C}$ and $650^{\circ} \mathrm{C}$ dislocations.

The following conclusions can be drawn from this EBIC work regarding the cause of the electrical activity observed at deformation induced dislocations.

1) High stress deformation at $420^{\circ} \mathrm{C}$ produces a small concentration of deep defect states, $>0.53 \mathrm{~V}$ below the conduction band edge. These states appear intrinsic to the dislocations and may be associated with some structural feature.

2) Copper decoration produces an extrinsic impurity level $0.48-0.51 \mathrm{~V}$ deep. For $420^{\circ} \mathrm{C}$ dislocations these states are above the Fermi level and hence do not contribute to recombination activity.

3) A shallow state, $0.34-0.37 \mathrm{~V}$ deep, is present at $650^{\circ} \mathrm{C}$ deformed dislocations. Its origin is unknown but may be due to residual impurities. It is lifted above the Fermi level when copper is added.

4) Dislocation kinks are sufficiently reconstructed so as to produce states no deeper than $\sim 0.3 \mathrm{~V}$ below the conduction band edge.

\section{EBIC Contrast From Decorated Oxidation Induced Stacking Faults in Silicon.}

In the previous section results were presented from well characterised dislocations where the recombination activity is believed to be due to states closely localised to the dislocation core. For this situation the model for recombination described above produces an excellent description of experimental results. However in many situations the defects to be studied are more complicated and it is expected that a different model of recombination is required. Figure 10a shows a TEM micrograph of clean steam oxidation induced stacking faults induced in p-type, low oxygen, CZ silicon. These defects were then decorated with either copper or iron. In each case the precipitation behaviour is markedly different and is shown in figure $10 \mathrm{~b}$ and c. Full details of these specimens and the precipitation observed may be found in $[20,21]$. However it is clear from the figure that large portions of the $1 / 3<111>$ Frank partial dislocation bounding the stacking fault in the copper specimen are now decorated with copper precipitates in the form of copper colonies. By comparison, in the iron specimen there is no evidence for any iron precipitation at the bounding partial and instead the iron precipitates in complex defects independent from the pre-existing dislocations of the stacking fault. Figures 10 (d-f) show EBIC micrographs of similar specimens. In the clean specimens $<0.1 \%$ EBIC contrast could be detected from the stacking faults and their partials which thus appear electrically inactive. For the copper specimen contrast of up to $30-40 \%$ was measured using a $15 \mathrm{kV}$ beam. This large amount of recombination clearly cannot be modelled in the same way as for the deformation induced dislocations. Indeed it is believed that a significant proportion of this contrast is due not to recombination, but instead to a reduction in the electron hole pair generation rate. This is because carriers are not generated within the precipitates themselves which for some copper colonies can account for a significant proportion of the generation volume. Recombination in the case of copper colonies will clearly depend not only on the physics of the process of recombination at the precipitate/silicon interface but also on the dispersion of the precipitates and other defects. For example a large concentration of small precipitates will provide a larger interface area than a smaller concentration of large precipitates of equal total volume. 
TEM
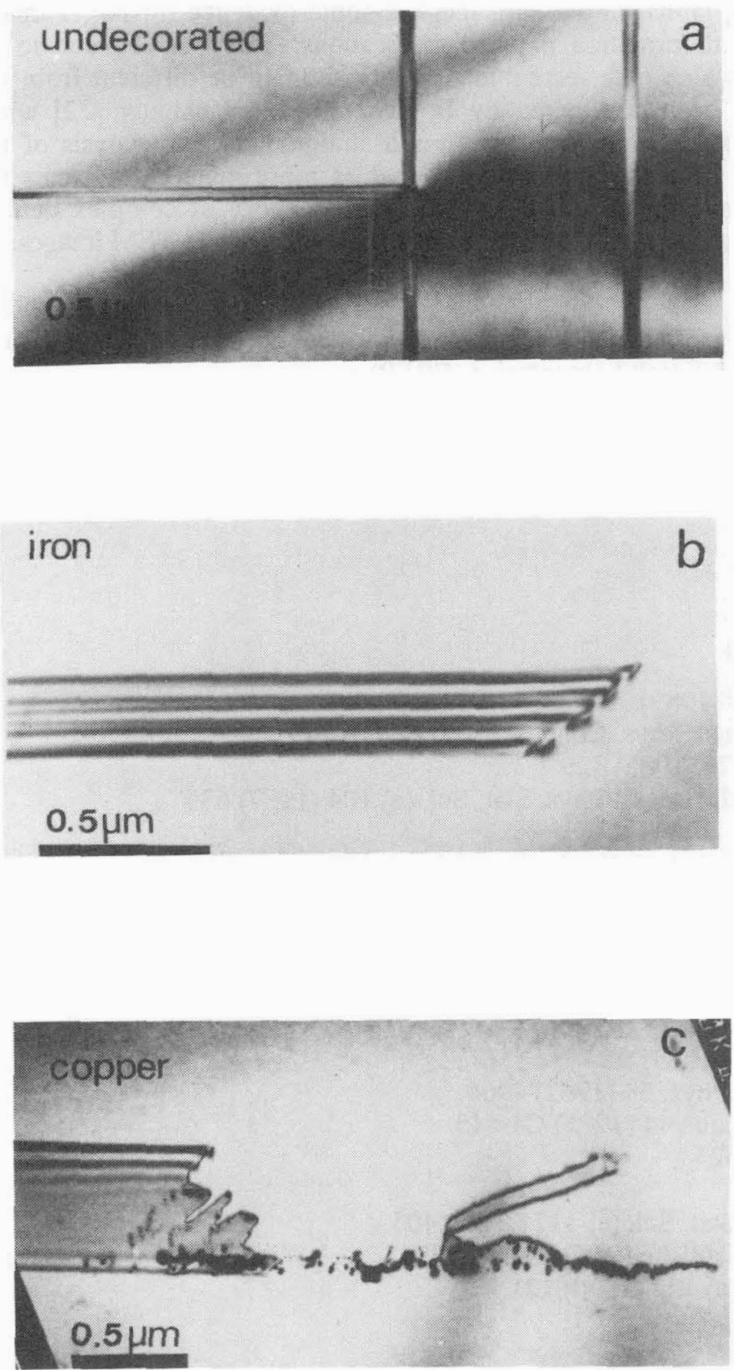

EBIC

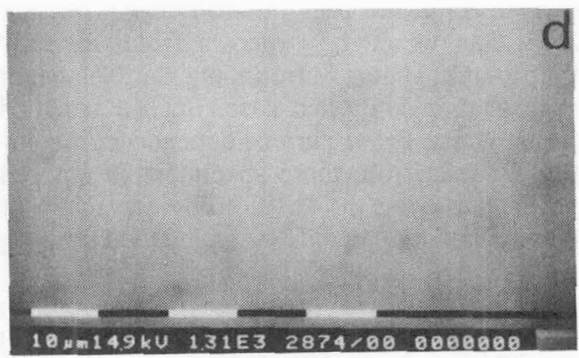

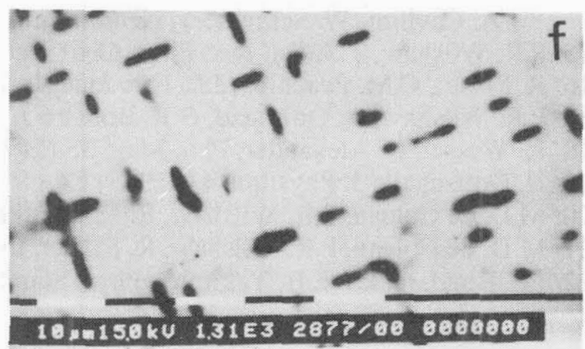

Figure 10. Micrographs of clean and decorated oxidation induced stacking faults in p-type silicon.

a) TEM micrograph of clean stacking faults. b) TEM micrograph of stacking faults from an iron contaminated specimen in which no precipitation was found on the bounding partial dislocations. c) TEM micrograph of a copper decorated stacking fault in which the bounding partial has served to nucleate a (111) copper colony. d) EBIC micrograph of clean oxidation induced stacking faults showing no electrical activity. e) EBIC micrograph of stacking faults in an iron contaminated specimen in which the stacking faults show electrical activity and the dark spots away from the stacking faults are due to recombination at iron precipitate/defect complexes. $f$ ) EBIC micrograph of a copper contaminated specimen showing the activity of the copper colonies. The EBIC amplifier settings were adjusted so that the contrast sensitivity is larger by a factor of two in image d) than e) and also a factor of two larger in e) than $\mathrm{f}$ ). 
The EBIC micrograph of the iron decorated specimen shows contrast of $\sim 18 \%$ from the bounding partials which from the TEM results is not due to the presence of decorating precipitates. The precipitate activity is seen as the small "dots" in the background between the stacking faults. In addition there is some evidence for larger image widths than would be expected from, for example, deformation induced dislocations. This may be due to an impurity atmosphere surrounding the dislocation in which case recombination would again be different from the purely charge controlled case. Such a situation has been considered by Bondarenko and Yakimov [22] when examining the beam current dependence of gold decorated dislocations. Thus a straight forward analysis of the EBIC contrast from these specimens is not possible. It is the authors' view that as more materials systems are investigated using the EBIC technique many more investigations will fall into this category of complex defects whose behaviour can only be understood with any degree of quantitative accuracy with reference to TEM images.

Acknowledgement: PRW gratefully acknowledges the financial support of the Royal Society, MDD gratefully acknowledges support from the Rhodes Trust and AT\&T Bell Laboratories (CRFP).

\section{References.}

/1/ H.J. Leamy, J. Appl. Phys. 53 (1982) R51

2/O. Breitenstein, J. Heydenreich, Scanning 7 (1985) 273

/3/ C. Donolato, Optik 52 (1978) 19

/4/ L. Pasemann, J. Appl. Phys. 69 (1991) 6387

15/ T. Wilson, E.M. McCabe, Optik 78 (1988) 59

/6/ C.E. Norman, D.B. Holt, Inst. Phys. Conf. Ser. 100 (1989) 731

గ/G. Weber, S. Dietrich, M. Huehne, H. Alexander, Inst. Phys. Conf. Ser. 100 (1989) 749

/8/ P.R. Wilshaw, G.R. Booker, Inst. Phys. Conf. Ser. 76 (1985) 329

/9/ A. Jakubowicz, H.-U. Harbermeier, A. Eisenbeiss, D. Kaess, Phys. Stat. Sol. (a) 104 (1987) 635

/10/ P.R. Wilshaw, Ultramicroscopy 31 (1989) 177

111/ A. Jakubowicz, Proc. 14 Int. Conf. Def. Semicond. (ed. H. Bardeleben) Mat. Sci. Forum 10-12 (1986) 475

/12/ H.K. Heinisch, Semiconductor Contacts, (International Series of Monographs on Physics) (Oxford University Press)

/13/ C.E. Norman, D,B. Holt, To be published in Inst. Phys. Conf. Ser. (1991)

14/ P.R. Wilshaw, T.S. Fell, G.R. Booker, Point and Extended Defects in Semiconductors (eds. G. Benedek, A. Cavllini, W. Schroeter) (Plenum Publishing Corp. 1989)

/15/ P.R. Wilshaw, T.S. Fell Inst. Phys. Conf. Ser. 104 (1989) 85

116/ R. Masut, C.M. Penchina, J.L. Farvaque, J. Appl. Phys. 53 (1982) 4964

/17/ P.R. Wilshaw, A. Ourmazd, G.R. Booker, J. Physique 44 (1983) C4-445

/18/ K. Wessel, H. Alexander, Phil. Mag. 35 (1977) 1523

/19/ H. Gottschalk, J. Physique 44 (1983) C4-69

/20/ M.D. de Coteau, P.R. Wilshaw, R. Falster, Phys. Stat. Sol. (a) 117 (1990) 403

21/ M.D. de Coteau, P.R. Wilshaw, R. Falster, to be published in GADEST'91

122/ I.E. Bondarenko, E.B. Yakimov, Phys. Stat. Sol. (a) 122 (1990) 121 